\title{
The 'Relay Ideation' Technique: Moving from Problem Understanding to Problem Solving in the Design Process
}

\author{
Karin Slegers ${ }^{1}$, Pieter Duysburgh ${ }^{2}$, and An Jacobs ${ }^{2}$ \\ ${ }^{1}$ CUO I Social Spaces, KU Leuven / iMinds, Parkstraat 45, bus 3605, 3000 Leuven, Belgium \\ 2 iMinds - Digital Society, SMIT - VUB, Pleinlaan 9, 1050 Brussels, Belgium \\ karin.slegers@soc.kuleuven.be, \\ \{Pieter.Duysburgh, An. Jacobs\} @vub.ac.be
}

\begin{abstract}
When describing the design process in product innovation, many authors identify phases that can be described as 'problem analysis' and 'generating ideas'. Several techniques are available to support design teams in each of these phases, but it remains a challenge to move from understanding a problem to coming up with ideas for concepts that might solve the problem. In addition, some of these techniques have counterproductive social side effects, which in fact may impede creativity in a design team. In this paper we describe a new technique for product idea generation called the 'relay ideation' technique. This technique was developed to help design teams move from understanding a problem to thinking creatively and concretely about the problem in order to generate concepts for innovative products or services. The technique is illustrated with a case study about IT applications for hearing-impaired children.
\end{abstract}

Keywords: Ideation, techniques, conceptualization, design team.

\section{Introduction}

Various authors have tried to capture and understand the creative process in design and have identified several steps in this process (for an overview see [1]). The majority of authors have identified two phases that can be understood as 'problem understanding' and 'problem solving'. In this respect, much has been written on involving targeted end-users and design teams in general in the design process (e.g. [2]), and particularly on how design concepts emerge from their sources of information (e.g. [3]). In addition, an abundant number of techniques has been developed and documented to support design teams in these phases of the design process (see [4] and [5] for some examples), many of which are variations on Osborn's 'brainstorming' technique [6]. In order to solve problems, this technique focuses on quantity, refrains from criticism, encourages unusual ideas and combines and improves ideas.

The transition from understanding a problem to solving it, which can be particularly challenging, can be understood as moving from openness to closure [7]. First, a design team needs to be open for ideas and views of others. Subsequently, it needs to draw conclusions and formulate solutions for observed needs and problems. Of course, in design thinking, 'a problem' and 'a solution' mean something different than 
in formal logic [8]. While in formal thinking a solution is either right or wrong, in design thinking this cannot be said: no answers can be considered 'correct'. In design thinking, solutions are to be understood as 'proposals', 'ideas', and 'suggestions'. Just as a 'the problem' is situational, and refers to the designer's current understanding of the situation, a 'solution' in design is temporal and always an ongoing process [8].

An additional challenge in the process of ideation and finding solutions, is the fact that design teams often struggle with group effects that are counterproductive for idea generation [1]. Theoretically, problem solving with a team should result in more creative solutions compared to an individual approach. But research has extensively shown that in practice the opposite is true. For instance, Warr and O'Neill [1] have identified three social influences on creativity that impede the theoretical benefits of group creativity: (a) production blocking, or the fact that verbally expressed ideas in a group allow only one person to express ideas at one time, while subsequently other team members may forget or suppress their ideas; (b) evaluation apprehension, or the fear of criticism of other team members; (c) free riding, or the phenomenon of group members becoming lazy and relying on other team members to contribute.

In this paper we present the relay ideation technique. With this technique we tried to find a means to help a design team (a) move from understanding to solving a problem and (b) overcome social group influences in the team that may impede creativity. Central to this technique is the concept of empathy. As the quality of design solutions is related to the empathy of a design team with the target group, the likelihood that products and services fit the end-users' lives well increases when these users and their experiences are well understood [9]. Therefore, before generating new ideas, a design team needs to gain empathy with the targeted user group, in order to understand the problem and to come up with relevant and useful solutions.

In what follows we explain the relay ideation technique. We further illustrate this technique by means of a practical research case. Finally, in the discussion, we will hint at some aspects of the technique we wish to explore further.

\section{$2 \quad$ Analysis and Ideation}

The relay ideation technique aims to facilitate the transition between the two phases in the creative process we mentioned above: problem analysis and problem solving.

\subsection{Problem Analysis}

It is assumed that before using the relay ideation technique, there has been a preparatory phase in which insights have been gathered about the needs and wants of targeted end-users, as is the common first step in a user-centered design process [10]. For the relay ideation technique, increasing empathy is seen as an essential step in analyzing the problem. In user-centered design, often only a few members of the design team (often design or social researchers) are directly involved in studying the needs and wants of the targeted end-users. However, when thinking about solutions for the users' problems, a larger group of people with various backgrounds is often involved 
(e.g. computer science, design, engineering, domain expertise). All members of the team need to gain empathy with the targeted users to find real solutions for them. In our view, simply communicating user insights to the design team is insufficient to let the design team think creatively about the needs and wants of the end users. Therefore, we recommend doing a team empathy exercise.

One example of such an empathy exercise is to have the design team create a number of what we refer to as 'empathy characters'. Although such empathy characters resemble personas that are often created in the design process to represent the problems and needs of the target group [11], they are not based on extensive ethnographic research and do not necessarily truthfully represent all characteristics and needs of the target group. Instead, empathy characters are a means to help members of the design team to empathize with the target group. In the empathy exercise, members of the design team bring a very basic character description to live by using user insights they were presented by the design or social researchers (we describe this process further in the case study in section 3). By doing this, the design team is encouraged to actively process the insights, which helps them to increase their understanding of the users. The team members discuss their empathy characters with each other and relate them to the project the team is working on. As such, several problems and challenges tend to come up that are relevant for the target group and for the project they are working on. A final step in the problem analysis phase is to select a number of these problems for the problem solving phase.

\subsection{Problem Solving: The Relay Ideation Technique}

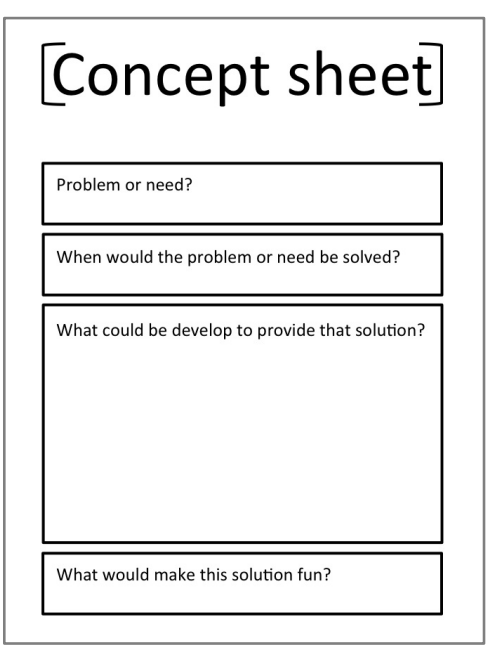

Fig. 1. The concept sheet

It is the problem solving phase that requires the design team to move from the problems and challenges analyzed previously to ideas for solutions. For this purpose, the relay ideation technique (illustrated in Fig. 1) encompasses an approach of working together in couples to 'solve' the problems selected in the problem analysis phase. This is facilitated by using socalled 'concept sheets'. These concept sheets consist of four empty fields that need to be completed in order to think of problem solutions in a stepwise fashion. The couples are not asked to fill in all the fields at once, but to complete only one field, and then pass the concept sheet on to the next couple. Ideally, there are at least as many couples as there are empty fields on the sheet.

In the first step of this problem solving procedure, each couple selects one of the problems that were listed in the problem analysis phase and describes more details of this problem in the first field of the concept sheet. After passing on the concept sheet to the next couple, each couple thinks of a 
general, abstract solution to the problem they were 'given' by another group. After passing on the concept sheets again, each couple formulates characteristics and functionalities of a concept that would provide this solution. Finally, after passing on the concept sheets one last time, the couples think of how to make the concept attractive.

Once all the fields of the concept sheets are completed, the exercise has resulted in a number of concepts equal to the amount of couples participating to the session (since every couple was given a concept sheet at the start). Each couple presents the concept they have finalized to the whole design team. The concepts can be further discussed, and others might add additional ideas or insights, and make adjustments to the concept. If time and attention allows, the exercise can be repeated.

\section{Case Study: Ideation for Hearing-Impaired Children}

We have used the relay ideation technique to generate new concepts for IT research projects in several contexts now, including assistive technology, music education and public transportation. To further illustrate the use of the relay ideation technique, we will briefly elaborate upon one of the projects in which we feel the technique was successfully used [12]. This project aimed at developing innovative IT concepts for hearing-impaired children, but besides that, the project had no specific technological focus at the start and was still in a very open, unfocused stage at the time the relay ideation technique was used. It was a collaborative project involving several academic partners. In the case study, two creative workshops were organized with the project design team, consisting of social researchers, designers and engineers. The workshops were moderated by the two social scientists who had also done the preparatory ethnographic research. The other team members had specific technical or design skills, but no knowledge of the targeted users.

\subsection{Problem Analysis: Empathy Exercise}

In the first half-day workshop, the two researchers who had done the ethnographic study communicated the main insights they had gained at a small poster 'exhibition'. The team was then subdivided into five couples and each couple was given an empathy character template. This template contained some basic demographic facts of the character the couples had to create, and four assignments that stimulated the couples to bring their character to life: (a) describe a typical day of this person (b) think of the most important problems and needs that this person might experience; (c) list this person's likes and dislikes in life; (d) describe possible dreams this person might have. For the basic demographics, four types of hearing-impaired children were included in the templates to reflect the diversity of the targeted users. Two characters were completely deaf; two were hard of hearing. One of the deaf children had a hearing family; the other had a deaf family. Two of the four children went to a special school; one of them lived at the school during the week.

By creating these empathy characters, all couples had to actively process the information they were presented with in the poster exhibition and they had to apply the 
information to the life of the particular character they were given. While filling in the template, the couples could ask additional questions to the moderators or consult the posters. When finished, the couples presented their characters to the entire design team. The moderators made use of this moment to correct some misunderstandings of the design team with respect to the insights that were first presented. Meanwhile, the problems and challenges that the characters were described to encounter in their lives were shared with all team members and listed on a flipchart.

The moderators felt that his empathy exercise was quite successful and helped the design team to better understand the targeted end-users. A problem that could be noted, however, was that a certain bias might result from repeatedly reinterpreting the original data. In all cases, the original data had been interpreted twice: once by the field researchers, and a second time by the design team. When a sign language interpreter had been involved in the ethnographic phase, the information from the hearingimpaired children had even been interpreted a third time. Even though the field researchers corrected some misunderstandings at the end of the character exercise, it is quite likely that their understanding of the target group was biased too.

\subsection{Problem Solving: Relay Ideation}

The aim of the second workshop, also a half-day workshop, with the design team was to generate ideas for new IT applications that might solve some of the problems that hearing-impaired children face with. This workshop took place in the same room as the first workshop. The posters of the exhibition, the empathy characters and the list of problems and needs were still in place and as such still visible to the team. The team was split up into four couples, this time for a relay ideation session.

During this relay ideation session, the concept sheets were used as described above. Each couple started by choosing and detailing one of the problems or needs listed on the flipchart in the first workshop. Then the concept sheets were passed on and the couples tried to describe a (non-technical) solution for the problem that was chosen by the previous couple. Again, the sheets were passed on and each couple tried to think of a concrete concept for a new IT application that might provide the solution described on the template they just received. Finally, after another exchange of concept sheets, each couple reflected on the solution and the concepts by indicating what elements would provide a fun experience for the children. As such, in one hour, 13 concepts were generated (one sheet included two concepts).

This process of circulating a step-by-step concept sheet turned out to be a thoughtprovoking technique. Because each couple had to think of different elements of the concepts (problem, solution, IT concept, fun elements), participants mentioned afterwards that the task never became boring. They felt continuously spurred and stimulated, because each couple constantly received surprising input from another couple. Neither was the exercise too difficult, as the couples did not have to think the whole solution through: they could just think of the next step and then literally handed the rest of the problem over to the following couple.

After the exercise with the concept sheets, the moderators added an additional task in which each couple tried to design a creative artifact made of scrap material, 
representing one of the concepts. To this aim, each couple chose one of the concepts that appealed most to them. By encouraging them to use creative materials, we wanted to stimulate the participants to 'think-by-doing', which affords unexpected realizations that one might not have arrived at without creating a concrete artifact (or prototype) [13]. As such, the couples became more critical of the concept and could reflect on it in more detail in terms of functionality, interaction, design, etc.

The concepts that resulted from the relay ideation approach in this project were validated in a workshop with technical project members and in two workshops with teachers of hearing-impaired children. Finally, three concepts were deemed to hold enough potential for further research and development.

\section{Discussion}

The relay ideation technique subdivides the transition from understanding a problem to solving a problem in small, incremental steps. By distributing the responsibility for solving the entire problem by passing on the concept sheet after finishing one single step of the problem solution process, we hoped to facilitate creative thinking. As such, the team members don't have to think the whole problem through at once. We wanted the relay ideation technique to be a productive technique: depending on the time reserved for each field on the concept sheet to be completed and the number of groups participating, the exercise should quickly result in a considerable amount of ideas. Finally, co-creating a concept may result in a feeling of shared ownership and support for the concept by the whole design team, which may in return increase the team involvement and the likelihood of further research and development on the concept.

This feeling of shared ownership might to a certain extent compensate for evaluation apprehension, one of the impeding social factors that has been linked to group brainstorms [1] that we mentioned in the introduction. As a result, criticism is less likely to be concentrated on a single person. Also, the fact that each field was completed by a couple, makes it less likely that a single person is to 'blame' for a step on the concept sheet. As the relay ideation technique allows for asynchronous working, it may also compensate for production blocking, another impeding social factor. Every couple is continuously busy, thinking about the field on the concept sheet they have in front of them. Furthermore, the fact that the team is split up into couples who have to pass on their work to the next team repeatedly, minimizes free riding, or the risk of any participant being lazy or relying on other team members too much. Working in small teams of two persons and (similar to the 'group passing technique' or the '6-35 ' method [5]) rotating the concept sheet creates a balance between the two opposing dynamics of restricting identification to avoid criticism and enabling identification to avoid the free riding effect suggested by Warr and O'Neill [1].

As with any idea-generating technique, the relay ideation technique does not guarantee high quality ideas. The quality depends on the participants, their inspiration, their motivation and many other determinants that are beyond the control of a technique. Therefore, the concepts that result from the relay ideation exercise should be 
regarded as 'raw materials', ideas that should be reviewed critically and will only to some extend be useful for the project.

The role of criticism in the ideation process has been a topic of much discussion. Osborn [6] believed criticism impedes creativity, and that it should be kept separate from thinking of new ideas. Warr and O'Neill [1] have also referred to fear of criticism as an impeding factor. However, research has shown that criticism is actually also essential to move beyond predictability and enhance creativity in idea generation [14]. For that reason, we included a moment to present and discuss the concepts that were generated at the end of the workshop. This can be done in a discussion, but also by, for instance, using scrap material, to make the concept more concrete and thus closer to reality (see e.g. [13] for more on engaging tangible working materials).

The relay ideation technique, in combination with the preparatory empathy exercise, is an attempt to stimulate group creativity by minimizing the effect of several contradictory social psychological effects. As we have not yet been able to validate the technique, in our future research we plan to experimentally compare the effectiveness of the techniques with that of other idea generation methods. In addition to this, we plan to study the experience of the members of the design team using the relay ideation technique into more detail.

Since its development, we have used the technique at several occasions. In future research projects we plan to develop and validate the technique further by experimentally testing which elements of the technique objectively result in better ideation processes. The experiences we have had with the technique so far already gave us several ideas to further alter and improve the technique:

(1) While an empathy exercise is an essential part of the relay ideation technique, there is not always time to allow for an elaborate process of context mapping and familiarizing the team with the end-users and their context. Further iterations of our technique should explore how this process can be more efficient, e.g., by including the character template in the concept sheet.

(2) Many models do not see creativity as a linear process [15]. However, our technique forces the design team to solve a problem in a linear manner. For new version of the relay ideation technique we'd like to explore possibilities to facilitate a nonlinear approach to problem solving. Exactly how this may be achieved is still unclear.

(3) Relay ideation can be used in several stages of the design process. In the case presented here, we used the technique in what is often described as the 'fuzzy front end' of design [2]. In later phases, the design team needs to take more issues and project decision into account. These restraints can influence the ideation process, resulting in different types of 'solutions', or requiring the design team to deal with the generated ideas in another manner.

In future research we hope to further develop the technique, which is still rather experimental and in that sense a work-in-progress, along these lines.

Acknowledgement. This work was part of the iMinds GR@SP project funded by iMinds (Interdisciplinary institute for Technology), a research institute founded by the Flemish Government. 


\section{References}

1. Warr, A., O’Neill, E.: Understanding design as a social creative process. In: Proceedings of the 5th Conference on Creativity \& Cognition, pp. 118-127. ACM, New York (2005)

2. Sanders, E.B.-N., Stappers, P.J.: Co-creation and the new landscapes of design. CoDesign 4, 5-18 (2008)

3. Halskov, K., Dalsgaard, P.: The emergence of ideas: the interplay between sources of inspiration and emerging design concepts. CoDesign 3, 185-211 (2007)

4. Herring, S.R., Jones, B.R., Bailey, B.P.: Idea Generation Techniques among Creative Professionals. In: 42nd Hawaii International Conference on System Sciences, HICSS 2009, pp. 1-10 (2009)

5. Bonneux, A., De Couvreur, L., Michiels, P., Vanneste, C., Verthé, B.: Innowiz, http: / /www. innowiz.be/index.php

6. Osborn, A.F.: Applied imagination: principles and procedures of creative problem-solving. Creative Education Foundation, Buffalo (1993)

7. Steen, M.: The fragility of human-centred design (2008), http: / / www . marcsteen.nl/texts.htm

8. Löwgren, J., Stolterman, E.: Thoughtful Interaction Design: A Design Perspective On Information Technology. MIT Press (2004)

9. Koskinen, I., Battarbee, K., Mattelmäki, T.: Empathic Design: User Experience in Product Design. IT Press (2003)

10. ISO/IEC: 9241-210:2010 - Ergonomics of human-system interaction - Part 210: Humancentred design for interactive systems, http: / / bit. ly/YEhJ0 5

11. Rosson, M.B., Carroll, J.M.: Usability engineering: scenario-based development of humancomputer interaction. Morgan-Kaufmann, New York (2002)

12. Slegers, K., Duysburgh, P.: Research Methods for Involving Hearing Impaired Children in IT innovation. In: Proceedings of NordiCHI, Reykjavik, Iceland, October 16-20, pp. 781784 (2010)

13. Agger Eriksen, M. Engaging design materials, formats and framings in specific, situated co-designing: A micro-material perspective. In: Online Proceedings of the Third Nordic Design Research Conference, Oslo, Norway (2009)

14. Nemeth, C.J., Personnaz, B., Personnaz, M., Goncalo, J.A.: The liberating role of conflict in group creativity: A study in two countries. European Journal of Social Psychology 34, 365-374 (2004)

15. Howard, T.J., Culley, S.J., Dekoninck, E.: Describing the creative design process by the integration of engineering design and cognitive psychology literature. Design Studies 29, $160-180$ (2008) 